\begin{tabular}{|c|c|c|}
\hline Région & glissements & $\begin{array}{l}\text { chûtes de pierres } \\
\text { ou éboulements }\end{array}$ \\
\hline . . . . & 40 & 4 \\
\hline Plateau . . . & 89 & 10 \\
\hline Alpes du côté $\mathbf{N}$ & 118 & 73 \\
\hline Alpes du côté $S$ & 154 & 56 \\
\hline
\end{tabular}

Dans la plupart des régions attaquées, la constitution géologique joue un rôle considérable. D'autre part, on a constaté que les accidents en question sont deux fois plus fréquents et nombreux dans les mois d'été qui sont très pluvieux, que pendant les mois des saisons fraiches et froides de l'hiver et du printemps. (Voir la figure: Diagramme).

\title{
ZUR GLIEDERUNG NEPALS IN NATUR- UND BEVÖLKERUNGSGEBIETE
}

\author{
Toni Hagen
}

Nepal war bis 1950 für Fremde ein praktisch verbotenes Land. Die wenigen Besucher, die von den damaligen Maharajas der Rana-Dynastie ein Einreisevisum erhielten, mußten sich auf die Hauptstadt beschränken. Einzig die Angehörigen der britischen Gesandtschaft hatten etwas Bewegungsfreiheit, die sie denn auch intensiv für botanische und zoologische Forschungen benutzten. Abgesehen von den in der Hauptstadt lebenden Newari war Nepal vom ethnologischen Standpunkt aus unbekannt.

Es blieb der Abteilung für Orientalistik der Londoner Universität vorbehalten, mit der systematischen Erforschung der ethnologischen Verhältnisse zu beginnen. Seit mehreren Jahren sind zahlreiche ihrer Kapazitäten in Nepal tätig, und mit großer Erwartung darf man ihren Veröffentlichungen entgegensehen.

Die folgende Abhandlung versucht eine erste Übersicht über die verschiedenen nepalischen Bevölkerungsgruppen und ihre Verteilung zu geben. Von Haus aus Geologe, hat es der Verfasser als seine Pflicht erachtet, auch den ethnologischen Belangen etwas nachzugehen. Hat doch bisher niemand außer ihm das gesamte Land bereist.

\section{NATURGEBIETE}

Nepal besitzt große landschaftliche Mannigfaltigkeit. Diese ist primär durch die starke vertikale Gliederung bedingt. Das nepalische Territorium reicht von der Gangesebene (200 m ü. M.) über die Hauptkette des Himalaya (über $8000 \mathrm{~m}$ ) bis in das Tibetische Hochplateau hinein $(4-5000 \mathrm{~m})$. Die Gegensätzlichkeit des Klimas bestimmen besonders zwei Faktoren: die Höhenunterschiede und die Lage zu Kontinent und Ozean, die sich in der ausgesprochenen Zweiteilung des Jahres in die feuchte Sommermonsun- und Trockenzeit äußert. Der Monsun dauert von Anfang Juni bis Ende September. Die achtmonatige Trockenzeit wird durch geringfügige gewittrige Niederschläge anfangs Januar etwas aufgelockert (sog. Wintermonsun).

Diesen Naturgrundlagen entspricht eine deutliche Gliederung von Nepal in sechs natürliche Großregionen :

1. der Terrai-Belt (= Sumpfstreifen), 2. die Siwaliks (entsprechend der Schweiz. Molasse am Alpenrand), 3. die Mahabharatkette (entsprechend der Alpenrandketten, Säntis z. B.), 4. die Midlands (Nepalisches Mittelland), 5. die Himalaya Hauptkette, 6. das Tibetische Plateau,

Mit Terrai-Belt wird der Anteil Nepals an der Gangesebene bezeichnet. Er liegt durchschnittlich $200 \mathrm{~m}$ ü. M. und bildet einen bis zu $45 \mathrm{~km}$ breiten Streifen von der indischen Grenze bis zum Fuß der Siwalikhügel. Östlich des Narayani Flusses allerdings folgt die indische Grenze dem südlichsten Kamm der Siwalikkette auf eine Länge von ca. $70 \mathrm{~km}$. Der Terrai-Belt ist zufolge der Sümpfe und des feucht-heißen Klimas eine wahre Fieberhölle. Trotzdem ist der waldlose 'Teil dicht bevölkert, vorwiegend mit Indern, die ihr Siedlungsgebiet schrittweise nach Norden vorschieben. Der Nordteil dagegen ist mit dichten Urwäldern bedeckt, in denen noch große Mengen von wilden Tieren hausen, wie Tiger, Leoparden, Braunbären, Büffel, Rhinozeros 


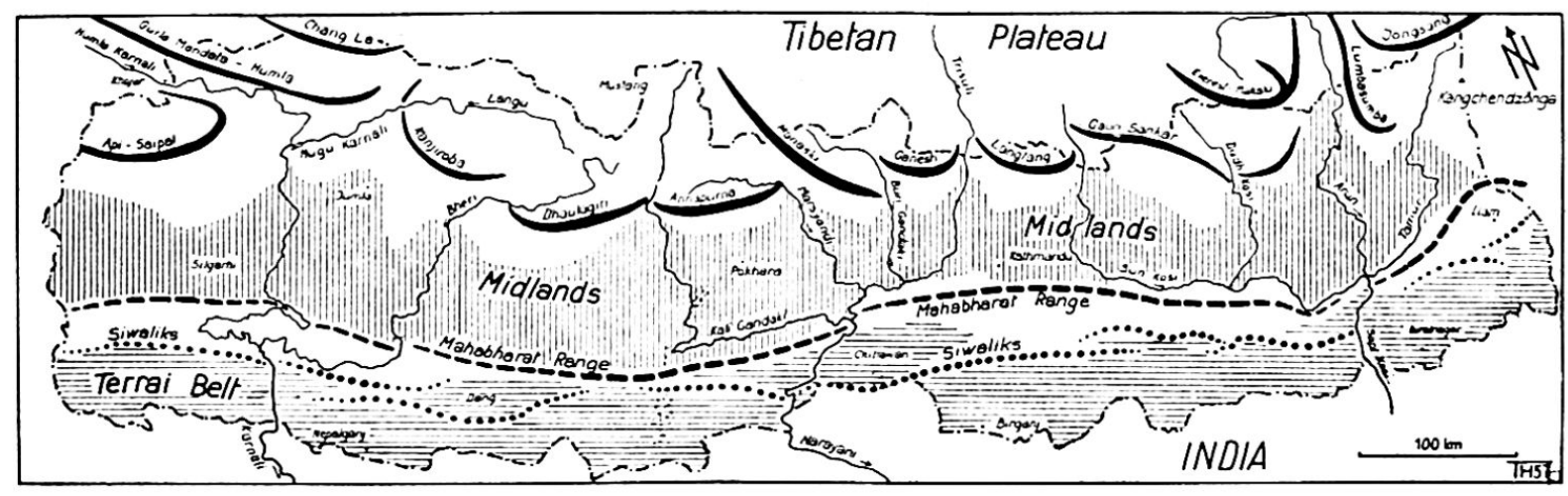

Main Himalaya Range

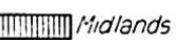

Mahacharal Range

Terrai Belr

Naturregionen von Nepal

und Elefanten. Die spärlichen Dörfer entsprechen Rodungsinseln im Urwald, und die Häuser sind zum Schutz vor den Überschwemmungen und vor den wilden Tieren teilweise als Pfahlbauten errichtet. Die nepalische Gebirgsbevölkerung meidet diese malariaverseuchten Sumpfgebiete vom April bis Oktober. Im Winter allerdings bewegt sich auf den Hauptrouten vom Nepalischen Mittelland her ein kontinuierlicher Strom von nepalischen Bauern, die zu Fuß ihre landwirtschaftlichen Produkte auf den indischen Markt bringen. (Auf der Tanakpur-Route z. B. wurden im Januar Morgens von 08.00-09.00 165 Nepali gezählt.)

Die Siwalikzone entspricht geologisch der gefalteten Molasse entlang dem Alpennordrand. Unvermittelt steigt sie aus der Gangesebene zu Höhen bis $1500 \mathrm{~m}$. Die Grenze Tiefland-Gebirge ist also deutlich linear. Die Siwalikzone besteht stellenweise

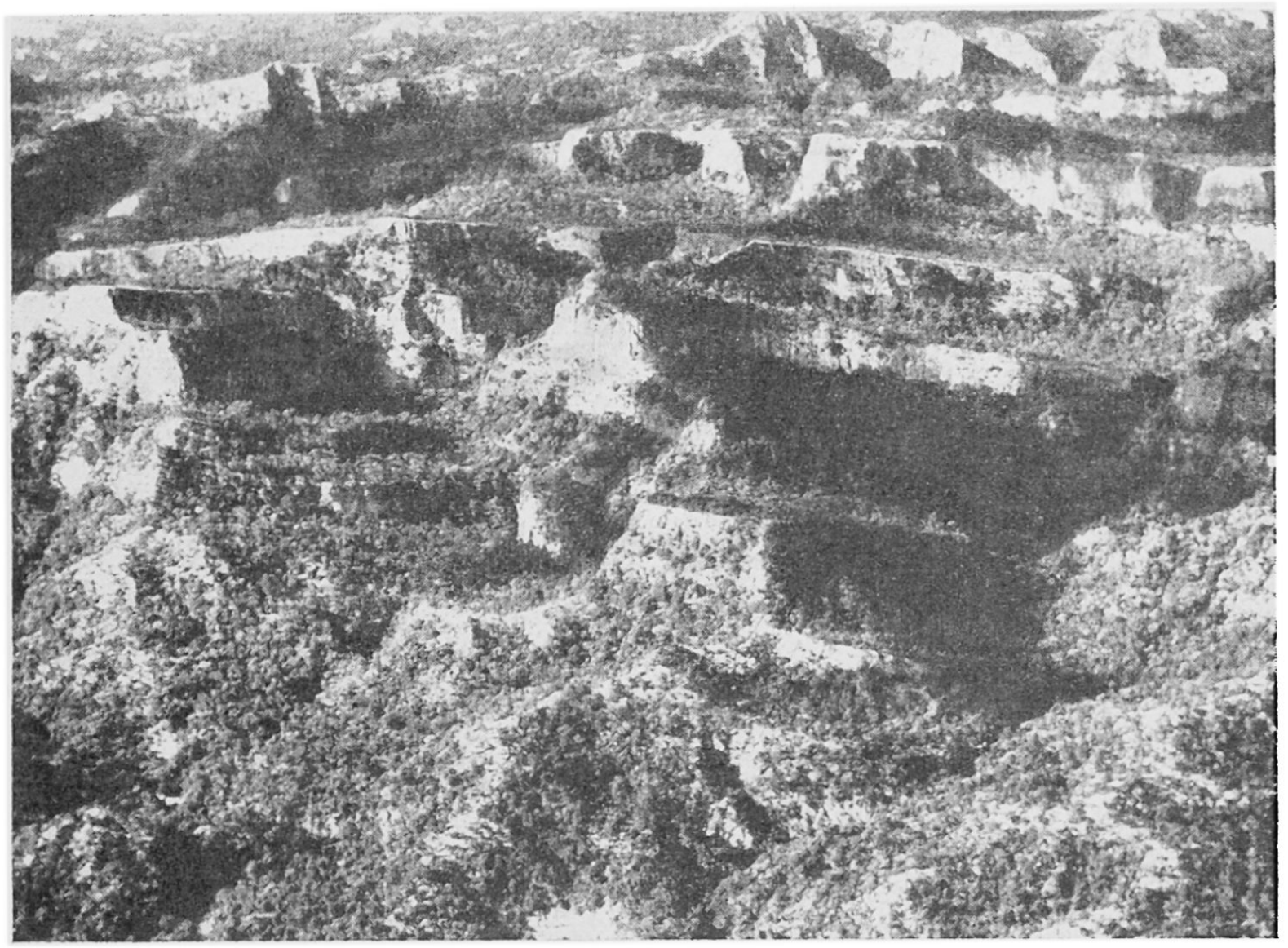

Landschaft der Siwaliks mit typischen urwaldverkleideten Sandsteinbänken. Alle Photos Dr. T. HAGEN 
aus einer einzigen Gebirgskette, welche sich an die nördlich anschließende, höhere Mahabharatkette anschmiegt. Vorwiegend nimmt sie aber einen breiteren Streifen ein, indem eine südliche, vorgelagerte Kette ein breites Talsystem (das sog. Dun) abtrennt. Die bekanntesten dieser Dun-Täler sind diejenigen von Chittawan und von Dang. Sie sind geologisch bedingt, indem dort die Faltungszone weit nach Süden ausgreift. Die Hügelzone der Siwaliks zeichnet sich durch wilde Zerklüftung aus. Sie macht, zusammen mit der Urwaldbedeckung die Region schwer begehbar. Wie der Terrai-Belt ist die Siwalikzone (die Dun-Täler eingeschlossen) nur dünn besiedelt. Dies ist nicht nur eine Folge der Verseuchung durch Malaria, sondern auch des geologisch bedingten, vorwiegend kargen Bodens, der hauptsächlich auf hellen, weichen, sehr glimmer- und quarzreichen Sandsteinen (Mittel-Siwaliks) und nagelfluhartigen, groben Konglomeraten (Ober-Siwaliks) beruht, welche zwar leicht verwittern, aber nur einen dürftigen Humus bilden. In weiten Gebieten liegen die groben Nagelfluhgerölle locker auf der Erdoberfläche. Es verwundert daher auch nicht, daß die ganze Siwalikzone wasserarm ist. Nur die Längstäler der Duns durchrinnen kleine Bäche.

Die Mahabharat-Kette ist bis $3000 \mathrm{~m}$ hoch. Geologisch gesehen bildet sie die Front der großen Überschiebungsdecken, die Brandungszone gegen die Siwalikzone. Sie gleicht weitgehend der entsprechenden Gebirgszone der Alpen, etwa dem Säntis oder Pilatus. Im Ganzen ist die Mahabharat-Kette eine große Synklinalzone, welche sich auf eine Länge von über $800 \mathrm{~km}$, von Garhwal bis Sikkim erstreckt. Sie besteht hauptsächlich aus den Kathmandu-Decken; nur zwischen Kali Gandaki und Karnali haben an ihr tektonische Einheiten der Nawakot- und Piuthan-Decken Anteil. Während die Kathmandu-Decken aus Gneisen, Glimmerschiefern und vereinzelten granitischen Einlagerungen bestehen, weisen die Nawakot- und Piuthan-Decken hauptsächlich Quarzite, Schiefer, Dolomite und Kalke auf. Auch die Mahabharat ist ein zerklüftetes Gebirge. Der Eindruck der Wildheit wird zwar durch die Vegetationsbedeckung (Buschwälder, Salhölzer, Rhododendron) etwas gemildert, aber die Talflanken sind ungewöhnlich steil. Die Bevölkerung ist demzufolge i. A. dünner als im Südterrai oder im Mittelland gesät. Nur in Gebieten, wo die Höhe des Hauptkammes nicht über $1500 \mathrm{~m}$ steigt (z. B. bei Tansing), finden sich größere Siedlungen. Auf einer Länge von $800 \mathrm{~km}$ durchbrechen große Himalayaflüsse nur an vier Stellen die Mahabharatkette, nämlich der Karnali, der Bheri, der Narayani und der Sapt Kosi.

Nördlich auf die Mahabharat-Kette folgt das Nepalische Mittelland (Midlands). Es bedeckt einen durchschnittlich 40 bis $65 \mathrm{~km}$ breiten Streifen. Jeder Wanderer, der die Mahabharat-Kette von Süden kommend überschreitet, ist überrascht, im Norden ein viel tiefer gelegenes Land mit ausgesprochen weichen Formen $\mathrm{zu}$ finden. Das Nepalische Mittelland liegt in einer Höhe von nur 600 bis etwa $2000 \mathrm{~m}$. Es wird von den hier weiten Talbecken der vom Himalaya kommenden großen Flüsse und auch der lokalen Flüsse gebildet. Rund $40-45 \%$ der 9 Mio Köpfe betragenden Bevölkerung Nepals wohnen in diesem Gebiet. Es ist sehr fruchtbar, und in ihm gedeihen alle Früchte und Gemüse der subtropischen und gemäßigten Klimazonen. Auch ost-westlich läßt sich eine gewisse Gliederung erkennen, die die Flußsysteme bedingen. Die 11 größeren Flüsse, welche die Hauptkette des Himalaya von Norden her durchbrechen, vereinigen sich im Mittelland zu drei Systemen: der Humla-Karnali, der Mugu-Karnali und der Bheri zum Karnali (letzterer allerdings erst zwischen Mahabharat und Siwaliks); der Kali Gandaki, Marsyandi, Buri Gandaki und Trisuli zum Narayani; der Sun Kosi, Dudh Kosi, Arun und Tamur zum Sapt Kosi. Dabei fällt auf, wie manche dieser Flüsse auf langer Strecke am Nordfuß der MahabharatKette dieser parallel fließen (z. B. Kali Gandaki und Sun Kosi). Durch pleistozäne Ablagerungen im Kathmandu-Tal, welche nach Süden, also gegen die MahabharatKette zu etwa $250 \mathrm{~m}$ ansteigen, ist erwiesen, daß die Mahabharat-Kette in jüngster 


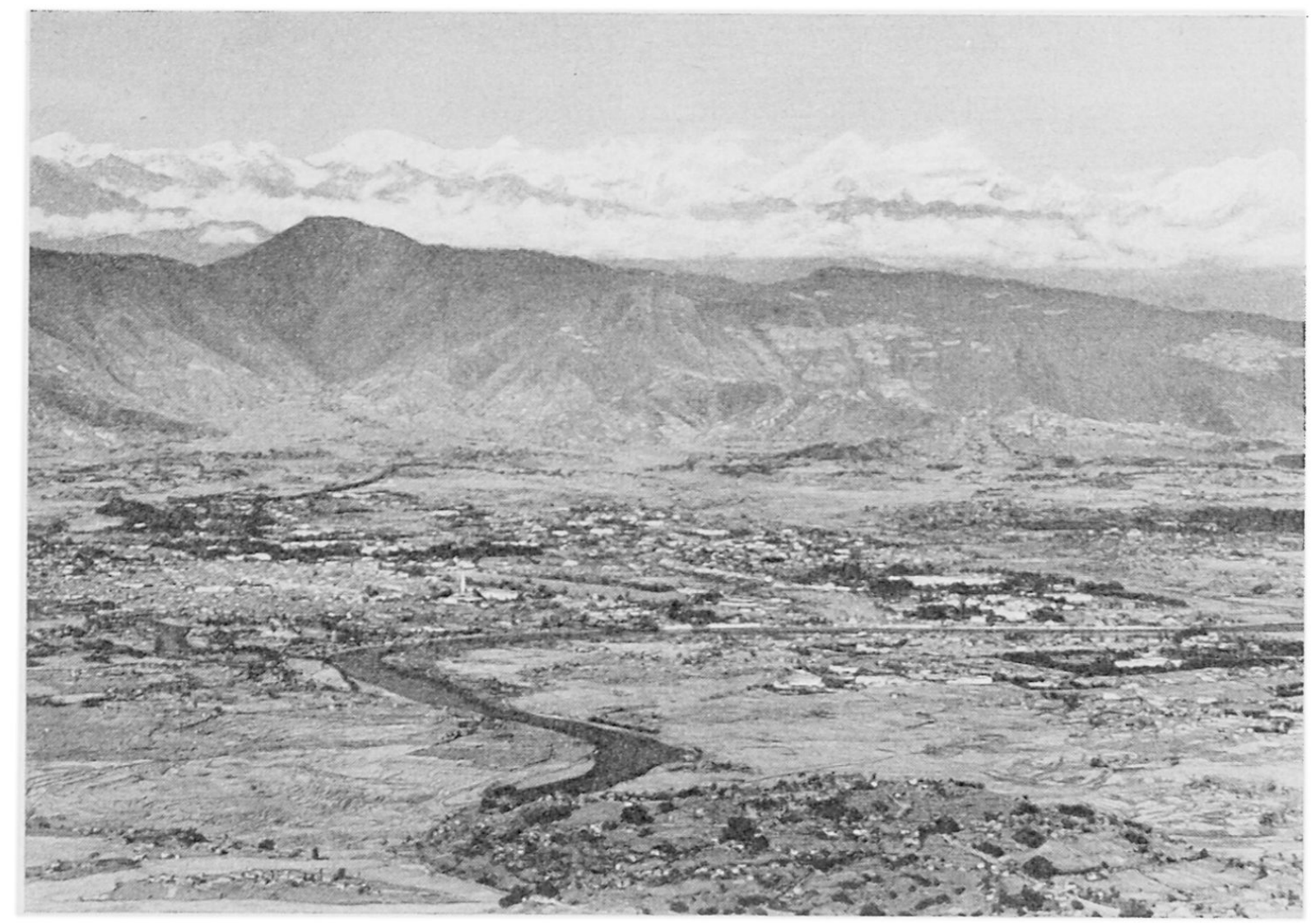

Kathmandu-Tal. Typische Landschaft des nepalesischen Mittellandes, ca. $1400 \mathrm{~m}$ ü. M. Im Vordergrund eine kleine Newarstadt inmitten ihrer Reisfelder.

Zeit relativ zum Mittelland gehoben worden ist, bzw. der Hauptgebirgskörper absank. Solche schiefgestellten Seeablagerungen (wohl pleistozänen Alters) wurden noch an mehreren anderen Stellen gefunden. Von diesen «Alpenrandseen» sind heute nur noch diejenigen von Pokhara vorhanden.

Die Hauptkette des Himalaya bildet im Ostteil des Landes die Grenze gegen 'Tibet (vom Jongsang Peak bis zum Langtang Himal). Westlich des Trisuliflusses reicht das Nepalische Territorium über die Hauptkette hinaus auf das Tibetische Plateau. Auch die Hauptkette gliedern die Querflüsse in einzelne Regionen. Sie ist indes, im Gegensatz zu anderen großen Gebirgen der Erde, nicht Wasserscheide. Diese liegt vielmehr weiter nördlich und erreicht nur bescheidene Höhen (bis höchstens $7000 \mathrm{~m}$ ). Sie trennt die Flußgebiete von Tsangpo (Brahmaputra) und Ganges. Des letztern Zuflüsse entspringen in ihr und durchbrechen die Hauptkette in gewaltigen Schluchten nach Süden.

Geologisch entspricht die Hauptkette des Himalaya in Nepal der steilgestellten Wurzelzone der großen alpinen Decken oder deren unmittelbar nördlich anschlieBenden Verschuppungen. Ihre einzelnen Segmente sind tektonisch bedingt, indem die Gebirgsgruppen den Schubbögen der Wurzeln entsprechen. Die Durchbruchsflüsse folgen durchwegs alten, voralpinen Querstörungen, welche aber während des alpinen Orogens reaktiviert worden sind. Die ausgeprägte Querstörung ist die riesige ArunQuerantiklinale, welche ca. $60 \mathrm{~km}$ nördlich der Hauptkette ihren Anfang nimmt, östlich des Kakalu eine Scheitelhöhe von mehreren $\mathrm{km}$ aufweist und mit letzten Ausläufern in Form von Brüchen und Verwerfungen sich bis zur Gangesebene, also auf eine Strecke von ca. $220 \mathrm{~km}$ !, bemerkbar macht.

Die nepalischen Gebiete nördlich des Himalaya zeigen vollständig tibetischen Charakter. Sie bilden eine Gebirgswüstenlandschaft zwischen 3000 und $5000 \mathrm{~m}$. 
Der Monsun wirkt sich hier kaum mehr aus: Der Verfasser erlebte, wie die Wolkenwand des Monsuns z. B. zwischen Dhaulagiri und Annapurna sich gleich einer Walze drehte, als Ganzes aber am selben Ort stehen blieb. Die Grenze der schweren Monsunregen liegt im Gebiet von Lete. 1-2 km nördlich davon fällt der Niederschlag - sofern die Wolken vorübergehend so weit gelangen - nur als feiner, kaum netzender Sprühregen. Die Winterniederschläge im Januar in Form von Schnee sind in der tibetischen Zone ausgiebiger. Trotzdem sind diese nördlichsten nepalischen Täler besiedelt. Man findet sogar Städte, wie z. B. Mustang $(3600 \mathrm{~m})$. Mit künstlicher Bewässerung gedeiht neben Kartoffeln vor allem Gerste. Daneben sind die ansässigen Tibeter intensiv mit Handel zwischen Tibet, Nepal und Indien beschäftigt.

\section{VERSUCH EINER ETHNOLOGISCHEN CHARAKTERISTIK}

Die ausgeschiedenen 6 Naturräume sind nicht alle gleichmäßig zur Bewirtschaftung und damit zur Besiedlung geeignet. Das Hochgebirge und die Siwalikzone (inklusive der Nordteil des urwaldbedeckten und malariaverseuchten Terrai) scheiden als Kulturzone zum vornherein aus. Im wesentlichen eignen sich nur drei der genannten Naturräume für die Landwirtschaft, der Süd-Terrai, das Mittelland und das Tibetische Plateau. Gemäß diesen drei Hauptzonen finden sich in Nepal denn auch drei Hauptgruppen von Völkern vor, die tibeto-birmanische, die tibetische und die indische.

Durch Wanderungen und Umschichtungen sind jedoch heute die drei genannten Hauptgruppen nicht mehr klar regional getrennt. Ihre Siedlungsgebiete sind mannigfach ineinander verzahnt.

Ursprünglich bewohnte die Tibetische Gruppe das Tibetische Plateau nördlich der Hauptkette des Himalaya. Die indische hatte ihre Heimat in Nordindien und im Süd-Terrai, während die tibeto-birmanische die eigentliche alteingesessene Urbevölkerung des nepalischen Mittellandes darstellt. Diese Hauptgruppen sind in zahlreiche Stämme gespalten, die sich nicht etwa nur durch verschiedene Dialekte, sondern durch verschiedene Sprache, Religion und Kultur voneinander unterscheiden. Alle haben eigene Bräuche, eigene Volkstänze, Kostüme, Feste; alle entwickelten eigene Haus- und Siedlungstypen und bearbeiten ihr Land nach besondern Methoden. Jedes Volk hat auch - gemäß der ihm eigenen Höhenlage — seine eigene Ernährungsweise.

Die im Gange befindlichen systematischen anthropologischen Untersuchungen werden gewiß den Beweis erbringen, daß mindestens ein Teil der verschiedenen Völker auch auf verschiedenen Rassen beruht. Bisher wurden hauptsächlich die Newars genauer untersucht (M. LOBSIGERDellenbach, 1952). Ich selbst habe zwar keine anthropologischen Messungen vorgenommen. Meine siebenjährigen Erfahrungen ermöglichten mir, die Nepali auf Grund ihres Körperbaues, Gesichtsausdruckes und ihrer Kleidung rein physiognomisch nicht nur der betreffenden Haupt-, sondern auch der Untergruppe zuzuteilen.

Die Tibeto-Birmanische Bevölkerungsgruppe bildet mutmaßlich die älteste eingesessene Bevölkerung des Nepalischen Mittellandes. Zu ihr gehören die Newari, Thamangs, Gurungs, Rais, Limbus, Mangars, Thakuris, Buras und Rukhas. Ihre regio-

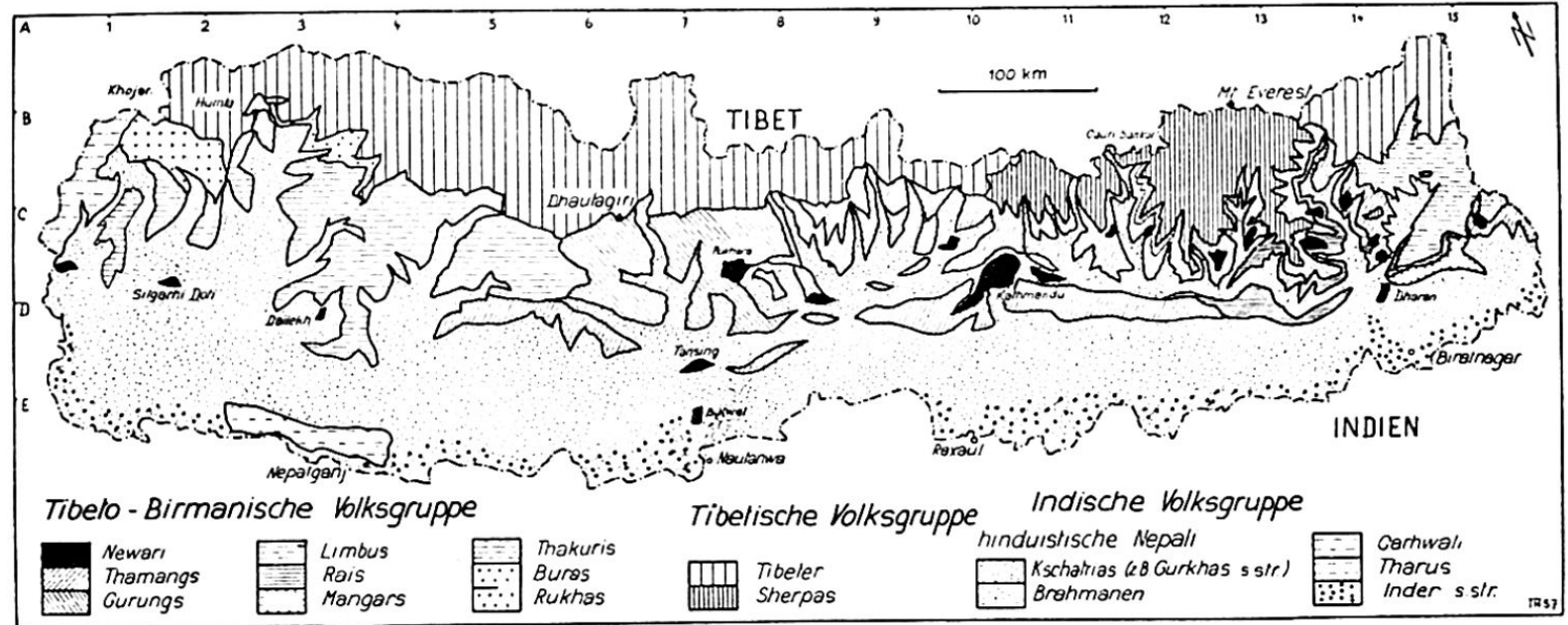

Bevölkerungsgruppen Nepals 


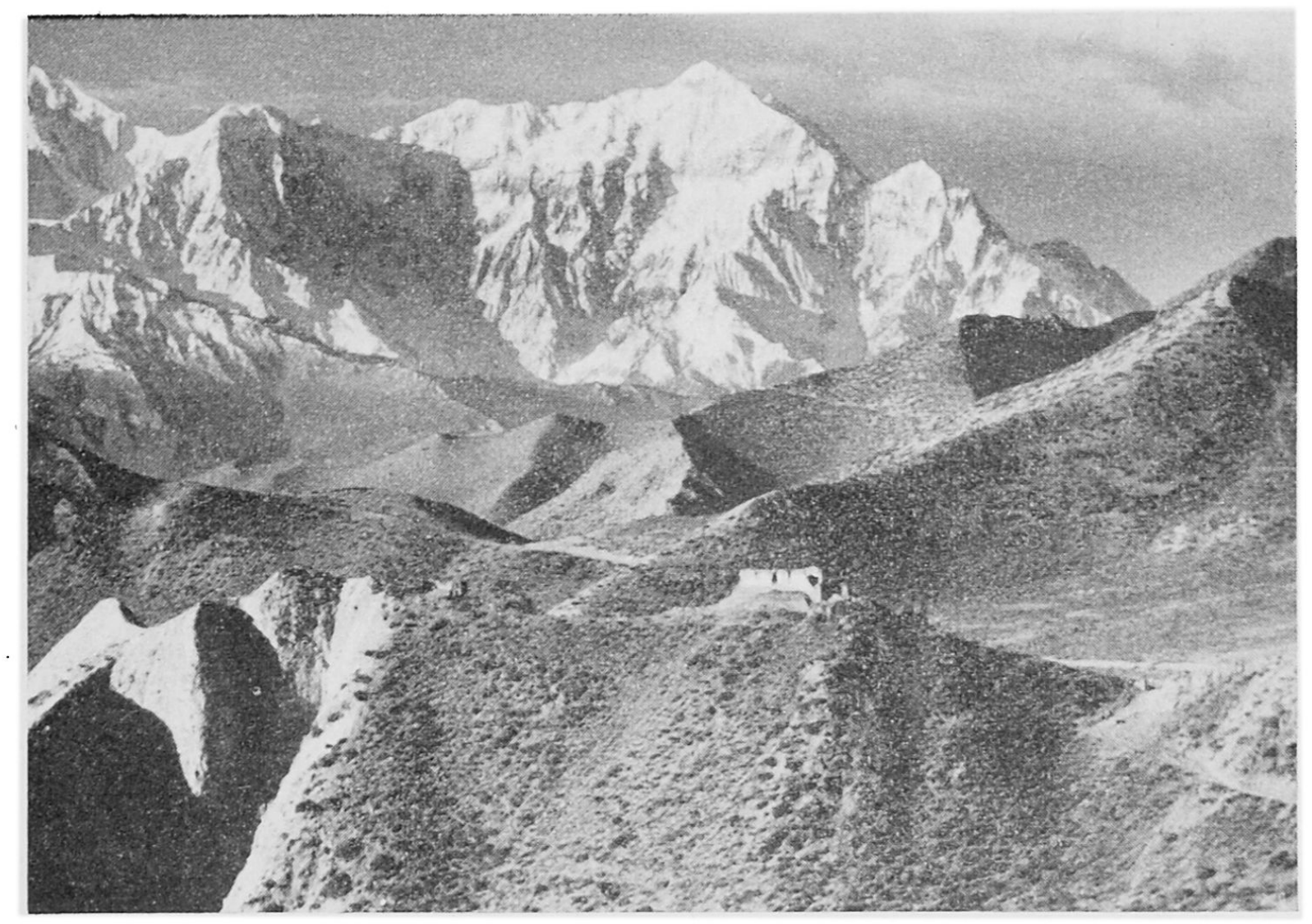

Landschaft des Tibetischen Plateaus; im Hintergrund die Annapurnakette von Norden

nale Verteilung ergibt ein ganz ungewöhnliches Bild. Die Siedlungsgebiete bilden keine zusammenhängende Flächen, sondern entsprechen der vertikalen Gliederung. Dies gilt ganz besonders für Ostnepal und für die Abgrenzung gegen die tibetische - und die tiefer siedelnde indische Volksgruppe. Volks- und Kulturgrenzen decken sich weitgehend mit gewissen Höhenkurven (was übrigens auch $\mathrm{C}_{\mathrm{H}}$. voN Fürer (1954) und H. Heuberger (1956) feststellten).

Die Newari sind die bedeutendste der ursprünglichen Gruppen; sie schufen die großen Städte Kathmandu, Patan, Bhadgaon und die reiche nepalische Kultur. Die Newari sind aber auch die nepalischen Handelsleute. Man findet sie demzufolge fast ausschließlich in Städten oder in Marktsiedlungen von kleinstädtischem Charakter. Letztere sind über das ganze Land verteilt, liegen aber vornehmlich an Verkehrsknotenpunkten, wo sich die lokalen Handelswege mit den großen Routen von Indien nach Tibet treffen. Die Newari waren ehemals Buddhisten - man trifft bei ihnen aber auch Reste der noch älteren vedischen Urreligionen -; sie sind jedoch zum größeren Teil zum Hinduismus übergetreten. Der Übertritt vollzog sich allmählich, wurde aber zeitweise durch die hinduistischen Malla-Könige beschleunigt. Im 14. Jahrhundert $\mathrm{z}$. B. drang die brahmanische Hierarchie im Kathmandu Tal ein, wobei die Buddhisten entsprechend ihren Berufen in Kasten eingeteilt wurden. Die Newars haben ihr Blut nicht rein erhalten. Ihrer leichtlebigen und toleranten Natur entsprechend wurden sie durch Jahrhunderte mit südindischem Blut durchsetzt. Ihre kulturelle Blüte erlebten die Newari - und damit Nepal überhaupt - im 15. bis 18 . Jahrhundert unter den berühmten Malla-Königen. Bis 1768 bestand das Gebiet des heutigen Nepal aus mehreren unabhängigen Königreichen und Fürstentümern. Die Malla-Königreiche wetteiferten miteinander im Bau von schönen Tempeln und in der Pflege von Dichtkunst und Musik. Ein Teil der heutigen Newari verrät mit ihren ausgeprägten Backenknochen und «Schlitzaugen» noch deutlich die Zugehörigkeit 
zur Tibeto-Birmanischen Volksgruppe. Auch ein «jüdischer» Typus mit breiten Nasen und starken Lippen ist vertreten. Daneben trifft man sehr schmale und feingliederige Typen, die auf Vermischung mit Indern hindeuten.

Die Thamangs bewohnen in der Regel die Gebiete über $1500 \mathrm{~m}$. Ihre größte Verbreitung haben sie in Ostnepal sowie in der Mahabharat-Kette südlich von Kathmandu. Der Glauben der Thamangs zeigt Anklänge an die vedische Religion, obschon sie heute weitgehend hindisiert sind. In der Nähe des Sherpagebietes findet sich ein besonderer Thamang'scher Lamaismus.

In Zentralnepal leben die Gurungs, an der Südflanke von Dhaulagiri und Annapurna. Auf den Höhenzügen haben sie sich vereinzelt bis fast zur Mahabharat-Kette angesiedelt. Die Gurungs besitzen die größten Dörfer Nepals. Ihre massiv gebauten Steinhäuser sind dicht aneinander gestellt und liegen vornehmlich an steilen Flanken, inmitten von Reisterrassen.

Die Rais bewohnten mittlere Höhenlagen in Ostnepal, zwischen Sun Kosi und Arun. Sie verraten am deutlichsten ihre birmanische Abstammung. Bei den Rais findet man außergewöhnlich schöne Frauen. Die Männer sind als Söldner berühmt geworden, haben sie sich doch in britischen Diensten den weitaus größten Prozentsatz von Viktoriakreuzen erworben. Sie sind ein festfreudiges Volk, und der Chang-Konsum (hausgemachtes Bier) nimmt ungewöhnliche Ausmaße an. Die Rais huldigen heute den Hindugöttern.

In den Tälern von Südostnepal, östlich des Arun sowie im Ostterrai leben die Mangars. Sie bevölkern nicht geschlossene Areale, sondern sind unter die andern Gruppen zerstreut. Ihr birmanischer Ursprung ist offenbar durch Vermischung mit In-

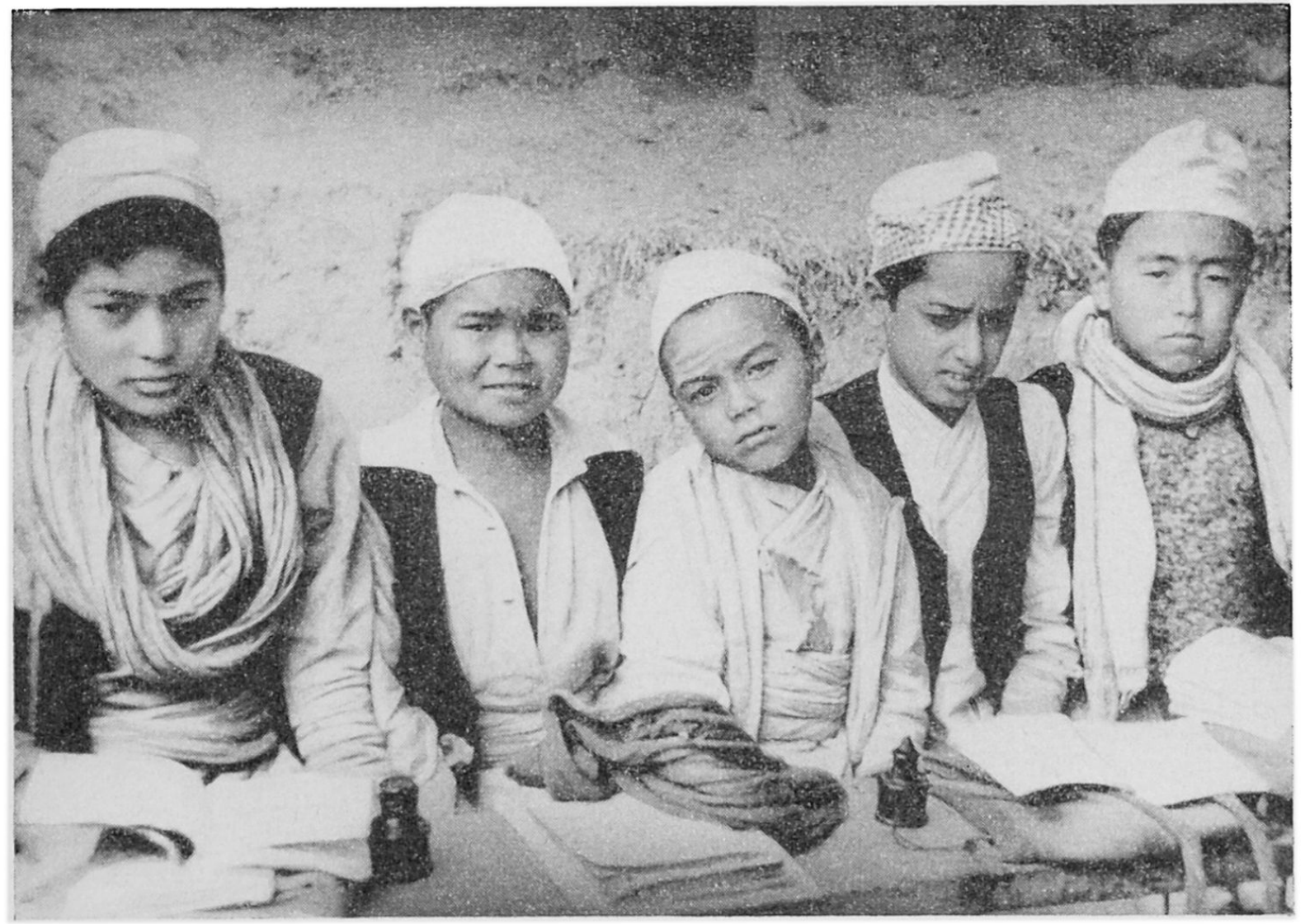

Typen in der Schule von Bhojpur (Ostnepal); von links nach rechts: Gami (Volksgruppe der Handwerker), Thamang (Tibeto-Birmanische Gruppe), Kschatria (Kriegerkaste der Hindus), Brahmane (Priesterkaste der Hindus), Rai (Birmanische Volksgruppe). Gemeinsame Unterrichtssprache ist das Nepali, auch Gurkhali genannt, die Sprache der Kschatrias. 


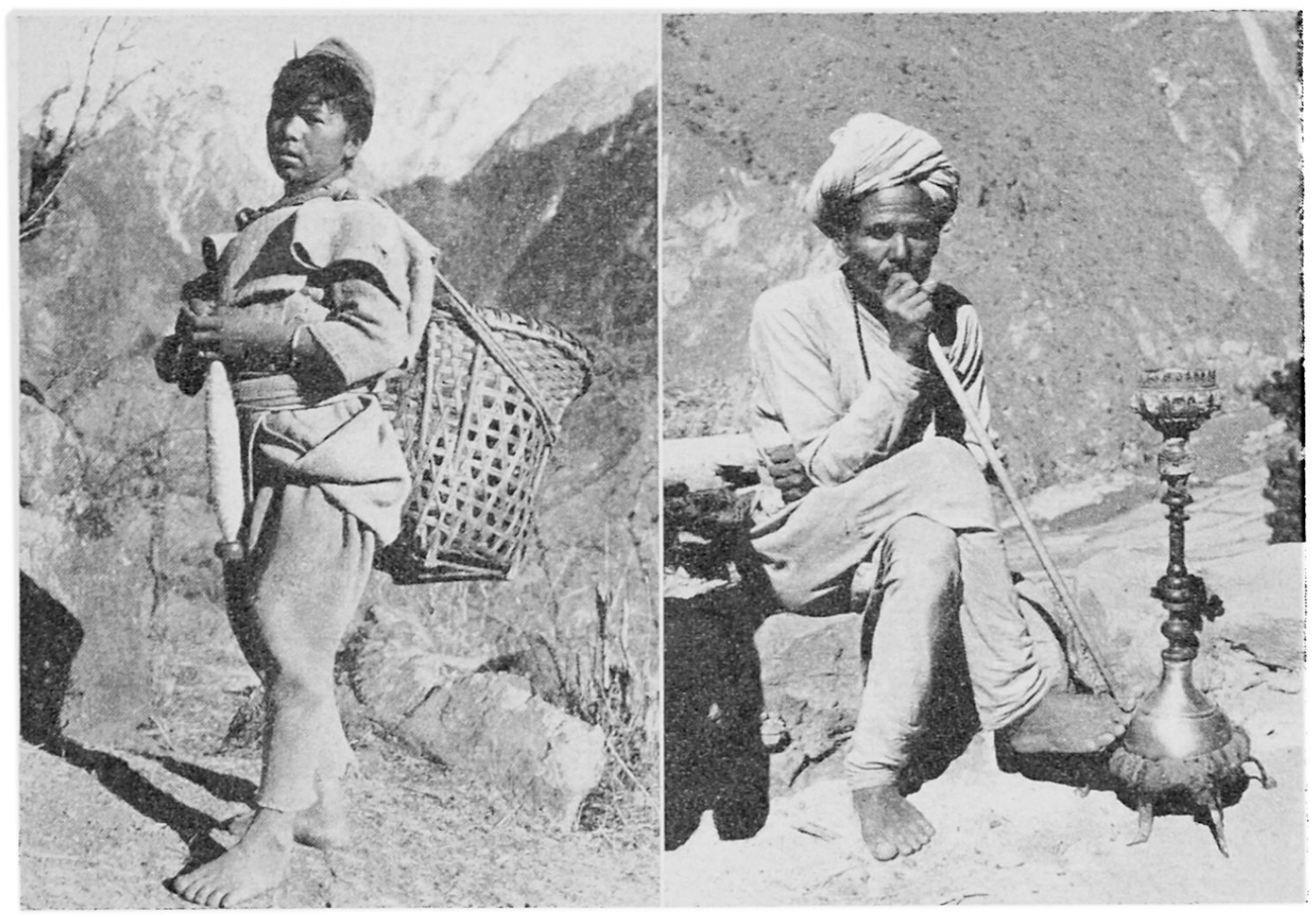

Links Rukha (Westnepal), Angehöriger einer kleinen tibeto-birmanischen Volksgruppe, Schafwolle spinnend. Rechts Thakuri mit der beliebten nepalischen Wasserpfeife.

dern stark verwischt. Man trifft sehr feingeschnittene Gesichter. Auch die Mangars sind Hinduisten.

Bei den Thakuris ist das sonst in Nepal herrschende Verbreitungsprinzip durchbrochen. Sie bewohnen nicht bestimmte Höhenlagen, sondern haben sich westlich des Kali Gandaki, vor allem zwischen Bheri und Karnali angesiedelt. Ursprünglich wohl tibetischen Ursprungs, opfern sie heute den Hindugöttern. Sie haben mongoloide Gesichtszüge bewahrt. Die Thakuris brachten den tibetischen Haustyp mit Flachdach sehr weit nach Süden, z. B. im Karnalital bis Raskot, d. h. bis ins nepalische Mittelland.

Die Buras und Rukhas sind kleine Volksgruppen, welche hauptsächlich Höhenlagen zwischen 2000 und $3000 \mathrm{~m}$ südlich, östlich und nordöstlich des Saipal in Nordwestnepal bewohnen. Es sind bisher fast unbekannte Völker. Es scheint, daß sie teilweise noch alten Religionen nachleben. Doch macht sich mehr und mehr der hinduistische Einfluß bemerkbar.

Die Tibeter bewohnen die Gebiete nördlich der Hauptkette des Himalaya. Es herrscht ein steter Bevölkerungsdruck von Norden her. Für die Tibeter mit den rauhen Wintern ihres Landes hat das warme und reiche Nepal die Bedeutung eines Paradieses. Es gibt denn auch nicht wenige tibetische Familien, welche die Winter im wärmeren Nepal verbringen, und sich mit dem Verkauf von mitgebrachten Fellen, Wolle und Halbedelsteinen durchbringen. Die Hauptkette des Himalaya mit ihren gewaltigen Schluchten wirkte indes lange als natürlicher Riegel. In Zentral- und Ostnepal, wo das Staatsgebiet weit nach Norden über die Hauptkette hinausgreift, verläuft die nepalisch-tibetische Kulturgrenze auf der Verbindungslinie der höchsten Gipfel. Die südlichst gelegenen Tibeter-Siedlungen in den einzelnen Durchbruchstälern finden sich wie folgt (Quarter inch map des Survey of India): Im Karnalital Kemri, Burong- 
sya und Dozam; im Mugu- Karnalital Chiti; im Bherital Pudamigaon, Ringmigaon und Tarakot (letzteres gemischt mit Thakuris); im Kali Gandakital Tukucha; im Marsyandital Thonje; im Buri Gandakital Ngyak und Thanju; im Trisulital Syabrubensi, im Bhote Kosi Tal Lapche Gömpa und Shoktra (beide in Tibet) sowie Lamobagar (mit Mischbevölkerung); im Sun Kosi Tal Khasa (in Tibet) ; im Aruntal Sempung. Östlich des Aruns, im Gebiet des Kangchendzönga, sind die Tibeter weiter nach Süden vorgedrungen. Ihre Siedlungen liegen dort weit südlich der Hauptkette, nämlich in Dongen im Mewatal; das sehr große Handelsdorf Walung im Tamurtal, und schließlich Khunsa und Yamphodin südlich des Kangchendzönga. Ursprünglich reichte auch der tibetische Buddhismus viel weiter nach Süden, denn heute kann man im rein hinduistischen Gebiet des Mittellandes (z. B. südlich Chauthara), noch alte, buddhistische Gebetsmauern sehen.

Die Sherpas sind ebenfalls tibetischen Ursprunges. Sie bewohnen die Südabdachung des Himalaya zwischen den Meridianen von Kathmandu und des Arun Flusses. Sie haben ihre eigene, jedoch mit dem Tibetischen verwandte Sprache, erhalten; als Buddhisten huldigen sie dem Lamaismus. Die Deutung des Namens Sherpa ist nicht abgeklärt; jedenfalls bezeichnen sie sich sogar in abgelegenen Gebirgstälern, welchc kaum je mit Expeditionen in Berührung gekommen sind, so. Sherpa bedeutet also nicht - wie fälschlicherweise landläufig immer wieder angenommen wird - «Träger»oder «Bergführer». Die Sherpas haben ihrer Heimat den Namen Solu Khumbu gegeben. Die darin zum Ausdruck kommende $Z$ weiteilung besteht auch tatsächlich geographisch. Das Gebiet oberhalb Chaunrikharka im Oberlauf des Dudh Kosi wird wie der Khumbu Gletscher am Mt. Everest «Khumbu» genannt. Das Khumbu ist ringsum von Hochgebirge umschlossen, vom Cho Oyu - Everest im Norden und von der Kwangde - Kangtega Gruppe im Süden. «Solu» (nach einem westlichen Seitenfluß. des Dudh Kosi) ist das «Unterland» der Sherpas. Das Khumbugebiet zeichnet sich durch große Dörfer, wie Namche Bazar (3300 m), Thami (3900 m) und Khumjung $(3900 \mathrm{~m})$ aus. Im Solu überwiegen Weiler und Einzelsiedlungen. Khumbu und Solu haben in den berühmten Klöstern von 'Thyangboche und Phaphlu ihre eigenen religiösen Zentren. Das Solu-Gebiet liegt an der Südabdachung des Himalayas. Von hier aus haben sich die Sherpas (immer über $2000 \mathrm{~m}$ ) noch weiter im Westen angesiedelt. Das westlichste Sherpazentrum ist Malemchigaon direkt nördlich von Kathmandu. Merkwürdigerweise unterstehen diese Gruppen dem tibetisch-buddhistischen Residenten von Nepal, dem Chini Lama, der seinen Sitz in Bodnath bei Kathmandu hat. Östlich des Solu, am Mayam Gebirge (Wasserscheide zwischen Dudh Kosi und Arun Fluß) finden sich ebenfalls noch vereinzelte Sherpasiedlungen. Über das Aruntal hinaus sind sie jedoch nicht mehr vorgestoßen, da dort die tibetische Bevölkerung von Sempung direkt an die hinduistische Kulturzone der Gurungs und Chetris grenzt. Auch sind dort in der hinduistischen Zone keine Spuren einer früheren Sherpakultur (z. B. Gebetsmauern) vorhanden. Vereinzelte Sherpasiedlungen finden sich schließlich noch entlang den Handelswegen vom Solu Khumbu nach Okhaldhunga - Indien und Darjeeling - Sikkim.

Aus dem Verbreitungsgebiet der Sherpas geht hervor, daß sie offenbar vor etwa einigen hundert Jahren von Tibet her über den Nangpa La (5500 m) und durch das Rongshartal nach Nepal eingewandert sind.

Die Sherpas sind vorwiegend Bauern. Die Solu Sherpas ziehen vor allem Gerste, Kartoffel und Mais. Die Khumbu Sherpas treiben daneben ausgedehnten Handel mit Tibet und Indien. Wichtigstes Handelsobjekt ist Salz, welches von Tibet her eingeführt wird. Die Sherpas versorgen auch einen großen Teil des südlich anschließenden Mittellandes mit Salz. Exportartikel nach Tibet (im Austausch gegen Salz) sind landwirtschaftliche Produkte, Papier und Petrol. Zum Transport nach Tibet werden Yaks verwendet. Viele der Khumbu Sherpas besitzen eine Art «Maiensäß» und Alpen in 


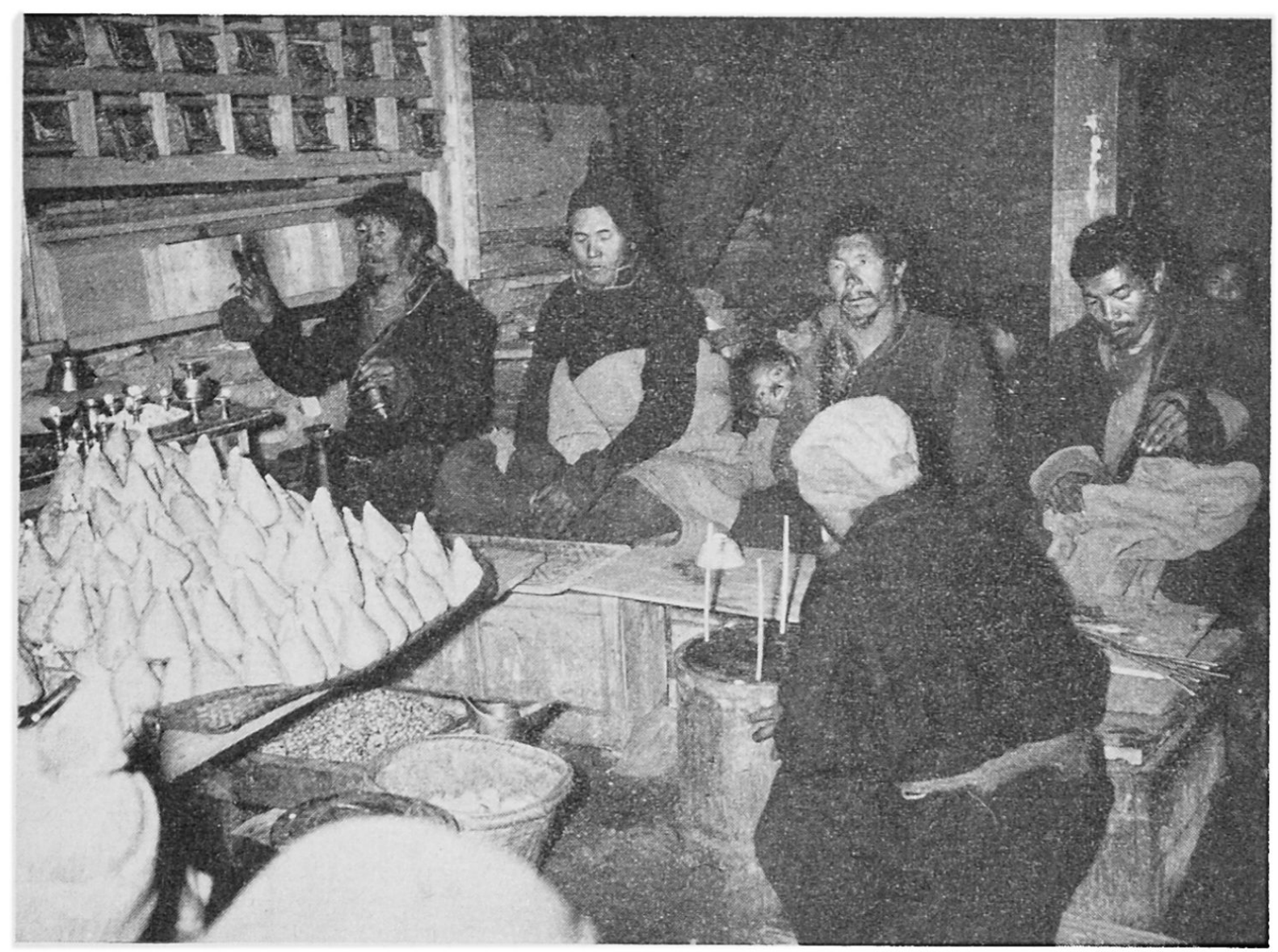

Sherpas, in einer Gömpa (Tempel) Reiskuchen und Bier (Chang) opfernd

mehreren Staffeln. Von allen nepalischen Volksgruppen haben die Sherpas die höchste Wohnkultur entwickelt. Sie besitzen ihren eigenen, zweistöckigen Haustyp mit flachem Giebeldach.

Besonders interessant ist die Grenze der Sherpas gegen die tiefer gelegenen hinduistischen Kulturzonen; sie entspricht teilweise geradezu den Höhenkurven, so derjenigen zwischen 2200 und $2400 \mathrm{~m}$. Diese Gliederung ist selbst auf einzelstehenden Vorgebirgen noch ausgeprägt, welche die $2200 \mathrm{~m}$ Grenze überragen. Man findet demzufolge auf solchen Vorgebirgen (z. B. am Mayam Gebirge) «insulare» Siedlungsgebiete der Sherpas, welche keine Verbindung mehr mit dem Hauptsiedlungsgebiet besitzen. Bei Wanderungen in diesen Gebieten, kann es geschehen, daß man am selben Tag mehrmals die Kulturgrenzen Sherpa - Thamang - Kschatrias (Hindus) und umgekehrt überschreitet.

Von der dritten Volksgruppe, der orthodox hincluistischen - oder arischen, wie man sie auch nennen könnte - sind nur die beiden obersten Kasten vertreten, nämlich die Brahmanen (Priesterkaste) und die Kschatrias (Kriegerkaste). Dies hat seine Ursache darin, daß diese Völker während der großen Religionskriege aus der nordindischen Tiefebene nach Nepal geflohen sind, und sich im dortigen sicheren Hügelland angesiedelt haben. Dabei folgten sie - als Tiefländer - in erster Linie den Talläufen. Dies erklärt das heutige merkwürdige Bild auf der ethnologischen Karte, auf der ihr Siedlungsgebiet fingerförmig in den Tälern weit nach Norden greift. Sie bewohnen heute die tieferen Zonen des Mittellandes, ungefähr unterhalb $1500-$ $1700 \mathrm{~m}$. Kschatrias und Brahmanen sind unter sich nicht nach verschiedene Höhenstufen getrennt, sondern bilden Dorf- oder Taleinheiten. Der erfahrene Nepalreisende erkennt sie sofort von den übrigen Rassen: Sie sind von schlankem, feingliederigem Wuchs und zeigen sehr feingeschnittene Gesichtszüge. Viele der Brahmanen zeichnen 
sich durch kühne Adlernase aus. Im Gegensatz zu den Tibeto-Birmanischen Rassen sind sie ernst, fast melancholisch und vermeiden Alkohol und Fleischgenuß.

Im allgemeinen lassen sich die Brahmanen und Kschatrias nicht mehr nach ihrer Herkunft aus Indien gliedern. Eine Ausnahme bildet der Stamm der ehemaligen radjputanischen Edelleute (Kschatrias), die sich nach ihrer Einwanderung zunächst gesamthaft im Gurkhagebiet in Zentralnepal angesiedelt haben. Diese erwiesen sich als äußerst dynamisch: Unter ihrem König Prittwi Narayan eroberten sie im Jahre 1768 die damals unabhängigen Königreiche der Mallas und andere kleine Provinz-Fürstentümer und begründeten das heutige Nepal. Ihre Sprache, nämlich das Gurkhali, hat sich zur allgemein verständlichen Nationalsprache entwickelt, zum Nepali. Dies ist in höchstem Maße erstaunlich, wenn man das Fehlen von jeglichen Verbindungsmitteln und Verkehrsmitteln sowie jeglicher Administration außerhalb der Hauptstadt Kathmandu berücksichtigt. Das Nepali wird heute fast im ganzen Land verstanden, obschon alle die verschiedenen Völker unter sich noch ihre eigenen Sprachen sprechen, die zum Teil wieder in verschiedene Dialekte zerfallen.

Der Begriff «Gurkha», wie er heute angewendet wird, sagt ethnologisch gar nichts, ja er ist geradezu irreführend. Die britische und die indische Armee rekrutieren seit langem die berühmten Soldaten aus Nepal, welche unter dem Sammelnamen «Gurkhas» bezeichnet worden sind. Jedoch beschränken sich die Nepalischen Söldner nicht nur auf die eigentlichen Kschatrias von Gurkha, sondern es wurden Männer aus fast allen Volksgruppen angeworben. Die Kschatrias von Gurkha nehmen dabei den kleineren Teil ein; die Hauptmasse rekrutiert sich aus den Gurungs, Rais, Thamangs und Limbus. Es wurden sogar Sherpas angeworben. Der Name Gurkha sollte demzufolge richtigerweise auf die Kschatrias von Gurkha beschränkt bleiben, zu denen die heutige Königsdynastie gehört.

Die Tharus, welche im Terrai-Belt nördlich Nepalganj leben, sind noch fast gar nicht bekannt. Es scheint, daß sie ebenfalls im Süden beheimatet sind.

In den südlichsten Zonen des Terrai Belt endlich haben sich reine Inder angesiedelt. Der Druck der Inder nach Norden (ethnologisch gesprochen) dauert an. Ihn üben neben der gewöhnlichen Landbevölkerung im südlichen Terrai namentlich indische Handelsleute aus, welche sich entlang den Haupthandelsrouten nach Norden ansiedeln. Aber auch die nepalischen Kschatrias und Brahmanen drücken die Thamangs und Gurungs nach Norden und in die Höhen: An vielen Stellen der von dichten Urwäldern bedeckten Talflanken am Südfuß des Himalaya sind neue Rodungen mit zahlreichen Siedlungen im Entstehen begriffen. Die obere Siedlungsgrenze an der Südabdachung des Himalaya (heute ca. $2300 \mathrm{~m}$ ) wandert allmählich in die Höhe.

$\mathrm{Da}$ anderseits aber auch die Tibeter und Sherpas einen Bevölkerungsdruck nach Süden und unten ausüben, geht dies auf Kosten der tibeto-birmanischen Urbevölkerung des Mittellandes. Es sind denn auch vornehmlich diese Gruppen, welche in großem Ausmaße aus Nepal auswandern. Man schätzt, daß ungefähr 2 Millionen Nepalesen (der Tibeto-Birmanischen Hügelrassen) in Indien leben. Dort sind sie sehr gesucht als zuverlässige Wachen für Banken, öffentliche Gebäude usw. Daneben ergießt sich noch jährlich ein Strom von hunderttausenden nepalischen Saisonarbeitern nach Indien, wo sie während der Winterzeit als Träger, Landarbeiter und Bauarbeiter etwas Bargeld verdienen.

Es wäre verlockend - und die gebildeten Nepalesen in Kathmandu tun es gerne die nepalische landschaftliche und folkloristische Vielgestaltigkeit mit derjenigen der Schweiz zu vergleichen. In der Schweiz sind die Talschaften als eigentliche Keimzellen unserer vielgestaltigen Kultur und Zivilisation bekannt. Das Volk einer Talschaft wehrte sich gemeinsam gegen die Naturgewalten, baute gemeinsam Wege und 
Brücken, Wasserleitungen und Schulhäuser. Es drängt sich die Frage auf: Warum ist Nepal heute das wohl am meisten «unterentwickelte» Land der Erde, obschon die Naturgrundlagen ähnlich sind wie in der Schweiz, und die handwerklichen Fähigkeiten der Nepali (wofür die prachtvollen Häuser und Tempel z. B. der Newari sprechen) sowie die menschlichen Qualitäten (wofür die weltberühmten Gurkhasoldaten mit ihrem Fleiß, ihrer Ehrlichkeit und Zuverlässigkeit sowie mit ihrem Mut Zeugnis ablegen) dem Schweizervolk in nichts nachstehen. Jeder Nepalkenner wundert sich immer wieder, daß die Nation Nepals überhaupt im heutigen Umfang besteht, wo doch kaum ein gemeinschaftlicher Geist vorhanden ist, abgesehen von einem (sich oft nachteilig auswirkenden) unbändigen Freiheitswillen. Die heterogene $\mathrm{Zu}$ sammensetzung des nepalischen Volkes, vor allem aber die außergewöhnliche regionale Verteilung der einzelnen Volksgruppen mag eine plausible Antwort geben: In Tälern, in welchen sich so grundsätzlich verschiedene Völker auf engem Raum je nach Höhenlage in die Besiedlung teilen, konnte gar kein Gemeinschaftsgefühl entstehen. Man findet denn auch Ansätze für Gemeinwesen bis jetzt nur in Gebieten, wo die Bevölkerung einheitlich ist, wie z. B. in den Newarstädten, oder in den hochgelegenen Tälern mit homogener Sherpa- oder Tibeter-Bevölkerung.

\section{WICHTIGSTE LITERATUR}

Fürer-Haimendorf, Ch. von (1954). Bei Bauernstämmen Ostnepals. Neue Zürcher Zeitung 19. 9. 1954. - Derselbe (1954). Besuch im Lande der Sherpas. Neue Zürcher Zeitung 17. 10. 1954. Heuberger, H. (1956). Der Weg zum Tscho Oyu. Mitt. d. Geograph. Ges. Wien, Bd. 98, Heft I. -Lobsiger-Dellenbach M. (1952). Himalaya du Népal. Mission scientifique Genevoise. Editions Jeheber, Genève - Paris. - Rauch, E.: Eindrücke eines Fluges über Nepal. Geographica Helvetica VIII, 1953, Nr. 4. - Derselbe: L’agriculture du Népal. Le Globe, 96, 1955.

\section{THE REGIONS AND POPULATIONS OF NEPAL}

The population of Nepal consists of at least 15 different groups. There are three main groups : the native Tibeto-Birman group of the Midlands, the Tibetan group of the Tibetan plateau and in the valleys in the main range of the Himalayas, and the Indian group, which has intruded from India. The Tibeto-Birman group consists of the Newars, Gurungs, Thamangs, Rais, Limbus, Thakuris, Mangars, Rukhas and Buras. The Sherpas and the pure Tibetans represent the Tibetan group, while the orthodox hinduistic group is composed of the highest casts only, the Brahmins and the Kschatrias (priests and warriors). All the above mentioned people have their own language (excepted Brahmins and Kschatrias), originally their own religion; further their own customs, celebrations, types of houses and settlements. Outstanding feature of the different people is their extraordinary distribution according to altitudes: The hinduistic group is living in the valleys, not higher than $2000 \mathrm{~m}$, the Tibeto-Birman group between $1500 \mathrm{~m}$ and $2400 \mathrm{~m}$ above sea level, while the Sherpas and the Tibetans are found above appr. $2200 \mathrm{~m}$.

\section{CARTE DE L'ÉCONOMIE MONDIALE}

\section{$1: 32000000$}

\section{Hans Boesch}

\section{QUELQUES REMARQUES ESSENTIELLES}

a) La carte de l'économie mondiale a été élaborée tout spécialement à l'usage des écoles secondaires et des gymnases. C'est la raison pour laquelle nous avons prié de nombreux professeurs de nous donner leur avis sur des cartes déjà existantes et de nous soumettre des suggestions pour une nouvelle carte. Il en est résulté une série de principes didactiques, dont il a fallu tenir compte. La carte à l'usage de l'enseignement doit avant tout donner des réponses claires et nettes à l'élève qui y cherche la solution des problèmes que lui pose le professeur. Une carte, si belle soit-elle, mais qui ne suffit

Editeur: Kümmerly \& FreY, Editions géographiques, Berne

Rédaction: Institut de Géographie de l'Université de Zurich, H. Boesch, Professeur, et A. Behrens, M. Muller, F. Casatello 